\title{
Measuring the Impact of Game Controllers on Player Experience in FPS Games
}

\author{
Kathrin M. Gerling, Matthias Klauser, Joerg Niesenhaus \\ University of Duisburg-Essen \\ Forsthausweg 2 \\ 47057 Duisburg, Germany \\ +49 (0) 2033791150 \\ \{kathrin.gerling, matthias.klauser, joerg.niesenhaus\}@uni-due.de
}

\begin{abstract}
An increasing amount of games is released on multiple platforms, and game designers face the challenge of integrating different interaction paradigms for console and $\mathrm{PC}$ users while keeping the core mechanics of a game. However, little research has addressed the influence of game controls on player experience. In this paper, we examine the impact of mouse and keyboard versus gamepad control in first-person shooters using the PC and PlayStation 3 versions of Battlefield: Bad Company 2. We conducted a study with 45 participants to compare player experience and game usability issues of participants who had previously played similar games on one of the respective gaming systems, while also exploring the effects of players being forced to switch to an unfamiliar platform. The results show that players switching to a new platform experience more usability issues and consider themselves more challenged, but report an equally positive overall experience as players on their comfort platform.
\end{abstract}

\section{Categories and Subject Descriptors}

H.5.2 [User Interfaces]: Ergonomics, Evaluation/methodology; K.8.0 [Personal Computing]: General - Game.

\section{General Terms}

Measurement, Experimentation, Human Factors.

\section{Keywords}

User experience, player experience, player engagement, usability, first person shooter, game controls, multi-platform development.

\section{INTRODUCTION}

Over the past years, multi-platform development has become increasingly popular in the video games industry. Many games are being simultaneously released for the PC, major console platforms as well as mobile devices. Besides technical limitations during the development process, different platforms are also associated with fundamentally different interaction paradigms or input control.

Permission to make digital or hard copies of all or part of this work for personal or classroom use is granted without fee provided that copies are not made or distributed for profit or commercial advantage and that copies bear this notice and the full citation on the first page. To copy otherwise, or republish, to post on servers or to redistribute to lists, requires prior specific permission and/or a fee.

MindTrek'11, September 28-30, 2011, Tampere, Finland.

Copyright 2011 ACM 978-1-4503-0816-8/11/09...\$10.00.
This is a major challenge for interaction designers since usable game controls have to be implemented without altering game mechanics - although sometimes the game design is adjusted for input controls and output rendering provided by the respective gaming system. Different input controls may also affect player experience, depending on how the available input device suits the game mechanics. This issue is not only important for player performance, but also for the individual, personal experience while engaging with games, largely referred to as user experience [1] or player experience [12]. With the recent interest in evaluating user experience in games, which lead to the development of questionnaires investigating player experiences, new survey measures are available to examine relations between hardware interface and player experience $[2,6]$.

In this paper, we aim to examine the impact of hardware game input controls on player experience. Because differences are likely to be especially prominent in games which rely heavily on mouse control, we present an evaluation of the first person shooting (FPS) game Battlefield: Bad Company 2. In our study, the game is either played on a regular PC using keyboard and mouse or on a Sony PlayStation 3 using the dual-shock 3 wireless game pad, because those two input controls represent the most common interaction paradigms for games of this genre.

\section{INTERACTION IN FPS GAMES}

\subsection{Control in PC and Console FPS Games}

When it comes to hand-eye coordination and reaction time, firstperson shooters are one of the most demanding game genres. The orientation of a player in a FPS game requires methods to control both the position and the direction of view of the player in the game world [8]. Early first-person shooters like Wolfenstein $3 D$ and Doom offer only horizontal orientation due to the lack of real $3 \mathrm{D}$ environments. Later first-person shooters added the possibility of changing the focus into all directions, a technique referred to as mouse-look [4]. Additionally, cursor keys were replaced by the WASD-keys for better ergonomic use of combined keyboard and mouse controls. This control scheme is still a common setup for PC-based FPS games. On consoles, FPSs had their breakthrough with Sony's PlayStation home console in 1994, which offered dual analog sticks. In most games, the left analog stick is used for forward and backward movement as well as strafing, while the right hand analogue stick is used for the free look in order to adjust the movement and fire direction, also many games offer additional setup options like an inverse z-axis. Although firstperson shooters are now a common sight on consoles and part of the biggest franchises of the gaming industry (Halo, Call of Duty, 
Killzone, Battlefield), critics often blame console controllers for their lack of precise game control. Also, the fact that a lot of console FPS games offer additional and/or optional player assistance like auto-locks and target snapping to overcome control issues suggests that console hardware is less suitable for FPS games than a keyboard/mouse setup on the PC.

\subsection{Studies of Player Performance in Games}

Natapov et al. [14] compared user's performances with Nintendo's Wii Remote and the Classic Controller for the task of pointing using Fitts's Law. A standard mouse was used as a baseline condition. In comparison to the mouse both console controllers performed poorly in terms of throughput, speed and error rate. A similar study by Klochek and MacKenzie [8] used a three-dimensional environment to compare an Xbox gamepad with a standard pc mouse for the tasks of tracking a moving target with and without acceleration over several seconds. While both tested devices performed equally well in tracking the target's velocity, the mouse allowed users to accelerate faster in order to correct errors in position. Isokoski and Martin [7] measured the performance of a wheel mouse, an Xbox360 gamepad, a keyboard with a standard mouse and a trackmouse in the task of FPS target acquisition. All settings using a mouse for aiming performed better than the Xbox360 gamepad. Another study developed a prototypical two-handed game input device for FPS games and compared a prototype with a standard mouse and joystick. Participants performed targeting tasks, scoring best with the experimental controller followed by mouse and joystick [9].

\section{PLAYER EXPERIENCE IN GAMES}

During the past years, different approaches towards describing and measuring player experience in games have been made. The term player experience is derived from the phenomenon of user experience (UX), which is defined in ISO 9241-210, describing how a person perceives and responds to the interaction with a system, highlighting the subjective, psychological nature of the phenomenon and focusing on the interaction process. Bernhaupt [1] underlines that player experience (PX) is dependent on subjective experiences evoked by games and phases of play they offer, and that interaction paradigms of games may influence PX.

\subsection{Models of Player Experience}

Player experience in video games describes the individual perception of the interaction process between player and game. Among others, the GameFlow model by Sweetser and Wyeth [17] describes player enjoyment in games based on Csikszentmihalyi's idea of Flow, which describes an experience evolving from the optimal alignment between challenge and personal abilities. They introduce dimensions such as challenge, skills or immersion, which are associated with criteria for enjoyment in games. Calvillo-Gámez [3] present the CEGE model, which aims to provide an instrument for the evaluation of gaming experience based on an examination of Core Elements of the Gaming Experience, Video Games and Puppetry. Similar to the GameFlow model, the CEGE model is based on an extensive analysis of psychological phenomena associated with player experience. Likewise, Poels et al. create a model of Nine UX Dimensions [16]. It is based on brainstorming sessions with players, which were held to gain insight into individual experiences while engaging with digital games. Additionally, expert reviews were introduced to further investigate aspects which might influence player experience. Thereby, nine dimensions of enjoyment in video games were identified, namely Flow, Imaginative and Sensory Immersion, Competence, Suspense, Negative Affect, Control and Social Presence. Based on these Nine UX Dimensions, the Game Experience Questionnaire [6] was developed, which we apply to measure PX in Battlefield: Bad Company 2. Further research on PX by Nacke and Drachen [12] highlights the temporal and contextual component of experience; for example, the impact of previous experience on PX which is especially important in the context of our study.

\subsection{Studies of Player Experience in Games}

Game metrics data is not only used for balancing and bugtracking, but also to define play-personas, which are modeled representations of how players interact with the game and can provide a deeper understanding of how players experience a game [18]. Questionnaires like the Game Experience Questionnaire (GEQ) [6] or the Game Engagement Questionnaire (GEnQ) [2] are used individually or are combined with other methods to measure player experience. Nacke \& Lindley [13] used questionnaires and psychophysiological methods to measure the player experience in a FPS game. In another empirical study [13], participants played a FPS game modification based on Half-Life 2 , in which sound and music were manipulated (on/off), while psychophysiological recordings of electrodermal activity (EDA) and facial muscle activity were recorded in addition to the Game Experience Questionnaire (GEQ). Results indicated no major impact on EMG and EDA, but showed significant effects on all dimensions of the GEQ. Nacke [11] compared the Wii Remote controls with a PS2 gamepad within the game Resident Evil 4 by measuring the psychophysiological responses, as well as spatial presence and the player experience using questionnaires. Although significantly different physiological results were found, game play was experienced equally well with both controllers. Likewise, Limperos et al. [10] explored the impact of Wii Remote and PS2 gamepad on player experience in Madden 2008, and found that the using the PS2 controller was subjectively perceived as more enjoyable. A study by Drachen et al. [5] indicates a significant correlation between psychophysiological arousal (i.e., EDA and heart rate) and self-reported gameplay experience (i.e., GEQ) while playing three different FPS games.

\section{EVALUATION: BFBC2 4.1 Battlefield: Bad Company 2}

Battlefield Bad Company 2 was released in 2010 by Electronic Arts and is one of the most popular first-person shooters on the PlayStation 3 and Xbox 360 consoles as well as the PC. It sold almost 6 million copies and has a metacritic score of 87 (PC) and 88 (PS3/Xbox360). The game offers solid and predictable single player mode and provides players with a typical single player campaign experience. BFBC2 uses common keyboard and mouse controls on the PC. On both consoles, the analog stick controls are consistent with the common control patterns of both sticks. Users can change the controls in the setup according to their preferences, yet during the study subjects were only allowed to inverse the z-axis.

\subsection{Methodology and Hypotheses}

We applied a $2 \times 2$ between subject design with the independent measure of participants being either comfortable with playing on a PC or on console platforms. To determine a participants' comfort platform, a short questionnaire on prior gaming experience was 


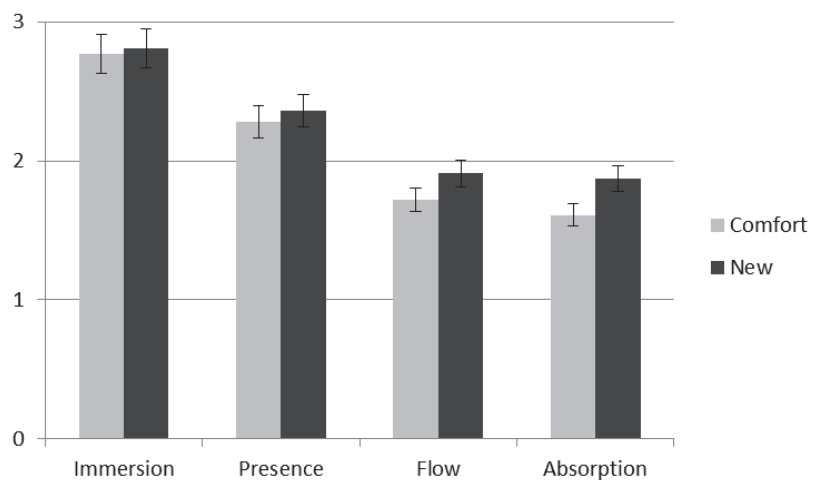

Figure 1. Mean GEnQ ratings for each platform (CI: 95\%).

applied. The dependent measures player experience, perceived usability and efficiency were collected as follows: The Game Experience Questionnaire (GEQ) first introduced by Ijsselsteijn et al. [6] measures the seven dimensions Competence, Flow, Negative Affect, Positive Affect, Challenge, Tension, and Immersion. Furthermore, we applied the Game Engagement Questionnaire (GEnQ) by Brockmyer et al. [2], which was designed to explore player engagement in video games and examines the dimensions Immersion, Presence, Flow and Absorption. ISO-Norm Questionnaire 9241/10 was included in the evaluation to investigate usability issues, which might potentially affect player experience. Additionally, we recorded basic player performance metrics, tracking how many times players died throughout the evaluation and investigating which parts of the level they completed. The following three main hypotheses are examined: The hardware interface influences the player's experience and efficiency (1), player efficiency has an impact on player experience (2) and using one's comfort platform will influence player experience (3).

\subsection{Participants and Procedure}

Forty-five adults participated in the evaluation $(\mathrm{N}=45,38$ male), their mean age was 24 years $(S D=3.5)$. Most participants were recruited at a local university. All subjects were familiar with FPS games, playing them on a regular basis using a $\mathrm{PC}$ or a gaming console (PS3 or Xbox 360), yet none of them had played BFBC2 before. After a short introduction to the experiment, participants were asked to provide information on previous gaming experience. Then, players were evenly assigned to one of the following four settings: (1) PC player assigned to PC condition, (2) PC player assigned to console condition, (3) console player assigned to console condition and (4) console player assigned to PC condition. Participants were asked to play the second level of the single player campaign of $\mathrm{BFBC} 2$, being told that their goal would be to reach the end of the level. Depending on the setting, either game pad or mouse and keyboard had to be used to accomplish this task. After twenty minutes, the session was aborted if players had not finished the level. Then, participants were asked to answer the GEQ, the GEnQ as well as the ISONorm questionnaire.

\subsection{Results}

The results of the evaluation suggest that the participants of this evaluation experienced an above-average gaming experience (cf. Figure 1 and Figure 2). Additionally, overall results of the ISONorm questionnaire suggest a low level of usability issues, yet ratings of perceived error tolerance and individualization were

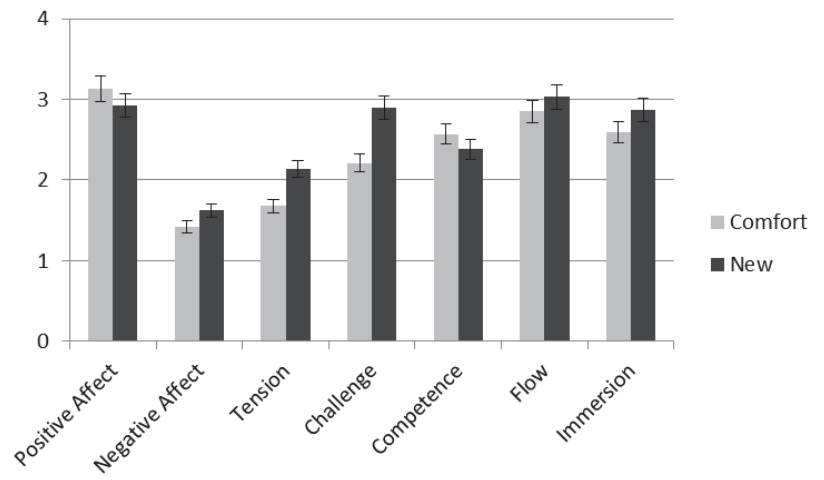

Figure 2. Mean GEQ ratings for each platform (CI: 95\%).

significantly better if players were using their comfort platform (cf. Table 1). Influence of the hardware interface (1): Three MANOVAs were calculated to examine if a particular hardware interface led to different levels of player experience, player's efficiency, or perceived usability. No significant results were found. Influence of efficiency on player experience (2): The number of deaths shows a positive correlation with the dimensions GEQ-Negative Affect $(\mathrm{r}=.428, \mathrm{p}=.004, \mathrm{~N}=43)$, GEQTension $(\mathrm{r}=.314, \mathrm{p}=.040, \mathrm{~N}=43)$ and GEQ-Challenge $(\mathrm{r}=.338$, $\mathrm{p}=.027, \mathrm{~N}=43$ ). Influence of the comfort platform (3): Three MANOVAs were calculated to examine if prior experience with the hardware interface led to different levels of player experience, efficiency, or perceived usability. For player experience, the following significant differences were found: Participants who did not use their comfortable device felt more challenged (GEQChallenge $\left(\mathrm{F}(41,1)=12.806 ; \mathrm{p}=.001, \eta_{\mathrm{p}}{ }^{2}=.238\right)$ than those who used their comfort device. Additionally, players felt more engaged (GEnQ-Absorption ( $\mathrm{F}(41,1)=5.017 ; \mathrm{p}=.031, \eta_{\mathrm{p}}^{2}=$ .109). Besides that, no significant results regarding player efficiency were found. However, differences in perceived usability were observed: Players using a new platform reported higher values for Error Tolerance $\left(\mathrm{F}(41,1)=8.502 ; \mathrm{p}=.006, \eta_{\mathrm{p}}{ }^{2}=\right.$ $.179)$ and on Individualization $\left(\mathrm{F}(41,1)=7.868 ; \mathrm{p}=.008, \eta_{\mathrm{p}}{ }^{2}=\right.$ $.168)$.

Table 1. Mean ratings for significant questionnaire results.

\begin{tabular}{lcccc}
\hline & \multicolumn{2}{c}{ Comfort Platform } & \multicolumn{2}{c}{ New Platform } \\
\cline { 2 - 5 } & $M$ & $S D$ & $M$ & $S D$ \\
\hline GEQ-Challenge & 2.21 & 0.59 & 2.90 & 0.68 \\
\hline GEnQ-Absorption & 1.61 & 0.39 & 1.87 & 0.39 \\
\hline Error Tolerance & 4.51 & 0.84 & 3.76 & 0.81 \\
\hline Individualization & 4.61 & 1.07 & 3.65 & 1.12 \\
\hline
\end{tabular}

\subsection{Discussion}

The results show that player experience in BFBC2 is only partially affected by gaming controller hardware. In this context, the most interesting observation is that although players felt significantly more challenged by the game if they were not allowed to play on their comfort platform, GEQ ratings for Positive Affect remained similarly high throughout all groups while Negative Affect ratings were relatively low. This suggests that if a certain level of usability is kept, a change in gaming platform is not followed by a significant decrease in player experience when engaging with $\mathrm{BFBC}$. This is supported by player performance metrics: Although player deaths seem to influence the ratings of the game in general, and players who die 
more frequently rate the game as more challenging, stressful and report a higher level of Negative Affect, no interrelations between comfort platform and this effect could be observed. However, perceived usability is affected by a platform change, as the results show that player ratings are significantly lower than when playing on a comfort platform. This issue may be caused by a lack of familiarity with interaction paradigms on different gaming platforms, or by users paying more attention to the interface as a result of being forced to play on an unfamiliar gaming platform.

However, the choice of controllers within this study bears certain limitations regarding its implications for the future development of multi-platform games: While designing for gamepad and keyboard/mouse setups represents one of the very basic problems in multi-platform game design, challenges associated with the recent focus on whole-body interaction in games (e.g. Microsoft Kinect and Sony PlayStation Move) are likely to affect games on a lower level. In this context, the implementation of controllerspecific game mechanics may be required to fully reveal the potential of motion-based gaming rather than mapping different control schemes to similar mechanics across multiple platforms.

\section{CONCLUSION AND FUTURE WORK}

In this paper, we briefly examined how player experience in first person shooter games is affected by the available gaming hardware using the example of Battlefield: Bad Company 2. The results show that although players do experience usability issues and feel more challenged when faced with a new gaming environment, their overall experience can be positive if the game is well-designed. Given the fact that BFBC2 is a rather popular game and reviews indicate a generally good gaming experience, this suggests that platform-related interaction problems have no significant impact on overall player enjoyment. Thus, rather than trying to further adjust FPS games to a particular platform, game design efforts could be focused on creating generally enjoyable game mechanics. Further research is necessary to establish common interaction paradigms, which might also support game designers when creating multi-platform games. In future work, we aim to examine interferences between gaming hardware and player experience using different gaming hardware. For example, impact of player experience on physical activity using novel game controls such as Microsoft Kinect. Additionally, the exploration of the impact of player experience in different game genres as well as the impact of different gaming platforms on less enjoyable games might provide further insight into the impact of controller hardware on player experience.

\section{ACKNOWLEDGMENTS}

We would like to thank Lennart Nacke, Jennifer Klatt and Oliver Daems for their feedback on this paper. Furthermore, we thank Tobias Reinartz and the participants of the evaluation for supporting our research.

\section{REFERENCES}

[1] Bernhaupt, R. 2010. User Experience Evaluation in Entertainment. In Evaluating User Experience in Games. Springer, London, UK.

[2] Brockmyer, J.H., Fox, C.M., Curtiss, K.A., McBroom, E., Burkhart, K.M., \& Pidruzny, J.N. 2009. The development of the Game Engagement Questionnaire: A measure of engagement in video game-playing. Journal of Experimental Social Psychology, 45, 624-634.
[3] Calvillo-Gámez, E. H., Cairns, P., \& Cox, A. L. 2010. Assessing the core elements of the gaming experience. In: Bernhaupt, R. (Ed.): Evaluating User Experience in Games. Springer, London, UK.

[4] Cummings, A.H. 2007. The Evolution of Game Controllers and Control Schemes and Their Effect on Their Games. In Proceedings of MC07, Southampton, UK.

[5] Drachen, A., Nacke, L.E., Yannakakis, G. \& Pedersen, A.L. 2010. Correlation Between Heart Rate, Electrodermal Activity and Player Experience in First-Person Shooter games. In Proc. of the $5^{\text {th }} A C M$ SIGGRAPH Symposium on Video Games. ACM, New York, NY, USA, 49-54.

[6] Ijsselsteijn, W.A., de Kort, Y.A.W., \& Poels, K. (in preparation). The Game Experience Questionnaire: Development of a self-report measure to assess the psychological impact of digital games.

[7] Isokoski, P. \& Martin, B. 2007. Performance of input devices in FPS target acquisition. In Proceedings of ACE 2007, ACM, New York, NY, USA, 240-241.

[8] Klochek, C. \& MacKenzie, I.S. 2006. Performance measures of game controllers in an three-dimensional environment. In Proceedings of Graphics Interface 2006, Toronto, Canada.

[9] Kwak, M. \& Salem, B. 2009. Designing a Game Controller for Novice HALO3 Players. In Proceedings of ICEC'09. Springer Verlag, Berlin, 325-326.

[10] Limperos, A.M., Schmierbach, M.G., Kegerise, A.D., \& Dardis, F.E. 2011. Gaming Across Different Consoles: Exploring the Influence of Control Scheme on Game-Player Enjoyment. Cyberpsychology, Behavior, and Social Networking, 14(6), 345-350.

[11] Nacke, L.E. 2010. Wiimote vs. Controller: Electroencephalographic Measurement of Affective Gameplay Interaction. In Proceedings of FuturePlay 2010, Vancouver, BC. 159-166.

[12] Nacke, L.E. \& Drachen, A. 2011. Towards a Framework of Player Experience Research. In Proceedings of EPEX'11 at FDG 2011, Bordeaux, France.

[13] Nacke, L.E. \& Lindley, C. 2009. Affective Ludology, Flow and Immersion in a FirstPerson Shooter: Measurement of Player Experience. Loading, 3(5).

[14] Napatov, D.,Castellucci, S.J., \& MacKenzie, I.S. 2009. ISO 9241-9 evaluation of video game controllers. In Proceedings of Graphics Interfaces 2009, Toronto, Canada.

[15] Pagulayan, R., Keeker, K., Wixon, D., Romero, R.L. \& Fuller, T. 2003. User-centered design in games. In: Jacko, J.A. and Sears, A. (Eds.) The Human-Computer Interaction Handbook, New York, USA: Lawrence Erlbaum Associates.

[16] Poels, K., de Kort, Y., \& Ijsselsteijn, W. 2007. "It is always a lot of fun!": Exploring Dimensions of Digital Dame Experience Using Focus Group Methodology. In Proceedings of FuturePlay 2007, Toronto, Canada.

[17] Sweetser, P. \& Wyeth, P. 2005. GameFlow: a model for evaluating player enjoyment in games. Computers in Entertainment, 3(3).

[18] Tychsen, A. \& Canossa, A. 2008. Defining Personas in Games Using Metrics. In Proceedings of FuturePlay 2008, Toronto, Canada. ACM, 2008, 73-80. 